\title{
Modified nasopharyngeal tube for upper airway obstruction
}

\author{
I B Masters, A B Chang, M Harris, M C O’Neil
}

\begin{abstract}
A modified nasopharyngeal tube is described that does not add airway dead space and resistance, is well tolerated, highly successful, and allows simultaneous use of oxygen prongs. This potentially reduces the need for surgical intervention to relieve high upper airway obstruction from Pierre-Robin syndrome and other causes.
\end{abstract}

(Arch Dis Child 1999;80:186-187)

Keywords: nasopharyngeal tube; upper airway obstruction; Pierre-Robin syndrome

Upper airway obstruction can cause hypoxia, hypercapnia, cor pulmonale, failure to thrive, gastro-oesophageal reflux, neurological impairment, and death. ${ }^{1}$ Methods used to relieve high upper airway obstruction proximal to the larynx include: placement on a modified bed, tracheostomy, ${ }^{2}$ and other surgical techniques (glossopexy, hyomandibulopexy, tongue-lip adhesion, circummandibular wire, and subperiosteal release of mouth floor ${ }^{134}$ ). Surgical techniques expose the infant to risks of anaesthesia and surgery and may not successfully relieve the upper airway obstruction. ${ }^{2}{ }^{4}$

The use of a nasopharyngeal tube to relieve high upper airway obstruction has been described in children with Pierre-Robin syndrome, ${ }^{35}$ but has not been uniformly successful. ${ }^{2}{ }^{6}$ We describe a novel way of relieving high upper airway obstruction with a modified, individually prepared, nasopharyngeal tube. Use of this nasopharyngeal tube potentially reduces the need for tracheostomy and other surgical intervention as it successfully relieves high upper airway obstruction that resolves with growth of the infant.

\section{Methods}

Medical records of children referred to our department with respiratory problems associated with high upper airway obstruction were reviewed; between 1994 and 1997 we managed all infants with a modified nasopharyngeal tube technique.

PREPARATION OF THE NASOPHARYNGEAL TUBE The required length of the tube is estimated (distance from lateral nostril to tragus of ipsilateral ear) and a tracheal tube placed in the nasopharynx, adjusted, temporarily taped, and its position radiologically assessed. In the ideal position, the nasopharyngeal tube tip sits just superior to the epiglottis and the obstructive breathing pattern is relieved. The tube length at the nostril is recorded when the correct position

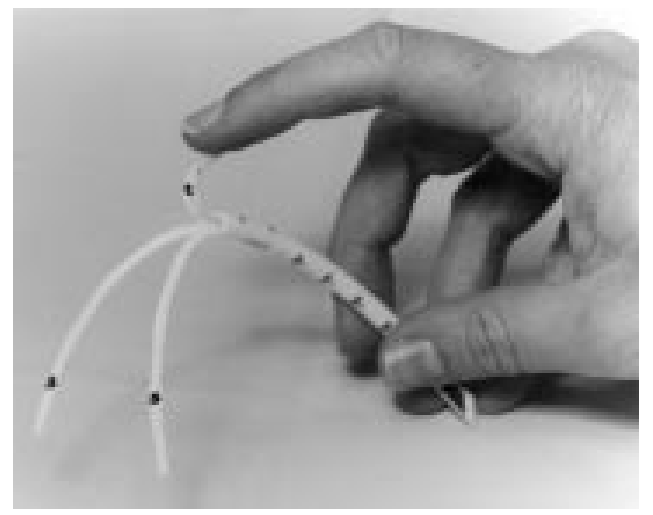

Figure 1 Prepared nasopharyngeal tube used for insertion into the right nasal passage. "s" are the side strips used for anchoring the tube to the infants cheeks. " $t$ " is the top strip used for anchoring the tube to the dorsum or lateral nose.

is established. A fresh tube (blue-line ivory tracheal tube, Portex, UK) is prepared by cutting at the measured length plus $5 \mathrm{~cm}$ (fig 1). The tube is then cut down the midline of the underside of the tube to the measured length. Two cuts are made to each side of the midline to create two thin strips $3 \mathrm{~mm}$ wide ("s" in fig 1 ), which are used for anchoring the tube to the child's cheeks. A further cut is made creating two additional strips on the top. One of these top strips (" $t$ "), shortened to the measured length plus $2 \mathrm{~cm}$, is used for anchoring the tube to the dorsum of the nose. The remaining top strip is cut off at the measured length. The wedge that is cut off depends on whether the nasopharyngeal tube is used for insertion into the right or left nasopharynx (fig 2).

\section{SECURING THE TUBE}

Before inserting the tube, a protective dressing (DuoDERM, Bristol-Myers, Montreal, Canada) approximately $4 \times 2.5 \mathrm{~cm}$ is applied to both cheeks and used as the base. The prepared nasopharyngeal tube is inserted into the selected nostril and the side strips "s" anchored on the base tape with adhesive tape. The smaller top strip " $\mathrm{t}$ " is passed across the ala and anchored on the dorsum or the lateral nasal aspect (fig 2).

In the initial period the nasopharyngeal tube size and length may require minor adjustments. The nasopharyngeal tube is changed every $2-4$ days for the first 10 days and then every 5-7 days thereafter; alternate nostrils are used. Parents are educated on tube changes, securing, and maintenance of the nasopharyngeal tube, including suctioning. It usually takes 5-10 minutes to change and secure the nasopharyngeal tube. Continued requirement for the nasopharyngeal tube was determined with overnight complex sleep polysomnography. ${ }^{1}$ 


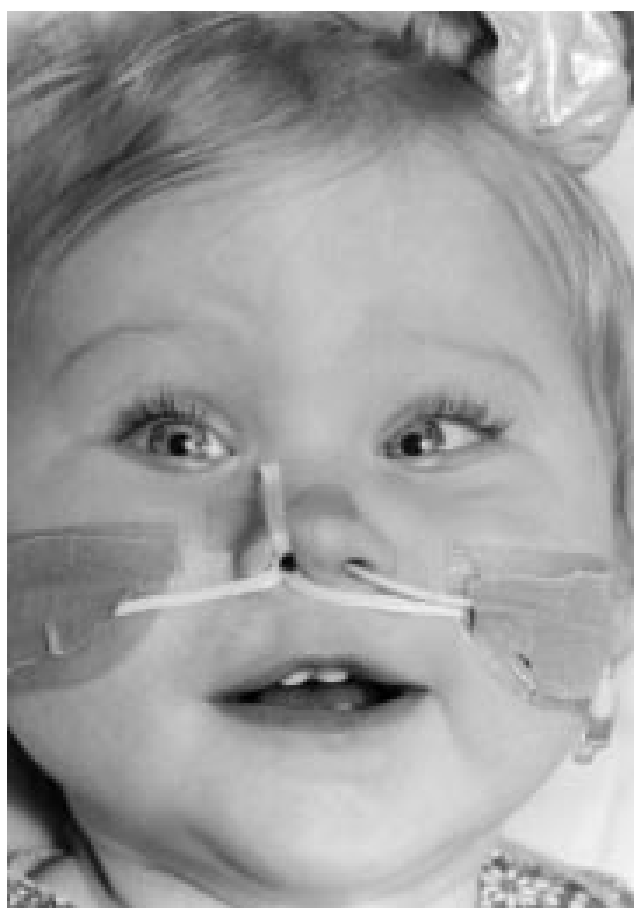

Figure 2 A child with a modified nasopharyngeal tube in situ in the right nostril and a nasogastric tube in the left.

The nasopharyngeal tube is still currently used in this child with idiopathic congenital hypotonia who started using it at 5 months of age. (Photo reproduced with permission of the child's parents.)

\section{Results}

The modified nasopharyngeal tube was used in nine infants (Pierre-Robin syndrome (6), isolated microngathia (1), Down's syndrome (1), idiopathic generalised hypotonia (1)). It was successful in all and surgical intervention was not required. Supplemental oxygen was used concurrently in four children (two as inpatients and two at home). Weight gain was significantly greater when nasopharyngeal tube was used (mean difference $169 \mathrm{~g} /$ week, 95\% confidence interval (CI) 103 to 233). In two children, supplemental feeds were required. In children with Pierre-Robin syndrome, the nasopharyngeal tube was required for a median of 6 months (range 5-7). In the children without Pierre-Robin syndrome, the tube is still in use in two children (range 6-15 months). In the initial period, three infants had regurgitation of feeds into the nasopharyngeal tube. No other complications occurred. A different size and/or length of tube was required for each nostril in four of the nine children.

\section{Discussion}

The importance of relieving upper airway obstruction has previously been highlighted..$^{15}$ Children managed with a nasopharyngeal tube had significantly better growth than those managed by sleep position. ${ }^{5}$ Children with Pierre-Robin syndrome may have normal intellect if recurrent hypoxic events are prevented. ${ }^{1}$

The traditional nasopharyngeal tube method used in children with Pierre-Robin syndrome is not always successful. ${ }^{2}{ }^{6}$ It requires labourious taping $^{5}$ and the length of the tube that protrudes from the nares adds dead space and airflow resistance. The connector makes the tube heavy, cumbersome, intrusive to the infant's face, and provides a handle for an older child to pull it out. We suspect these factors contribute to the failure of the traditional nasopharyngeal tube. The advantages of using the modified nasopharyngeal tube include: the ease of nasopharyngeal tube changes, absence of additional dead space and resistance, reduction in weight, more acceptable visual appearance, and the ability to use oxygen prongs simultaneously. Our nasopharyngeal tube changes are easier because of the absence of ties and bandaging. Our technique was successfully used in infants with high upper airway obstruction from causes other than PierreRobin syndrome.

This novel, modified nasopharyngeal tube technique to relieve high upper airway obstruction is preferable to surgical intervention as it is non-invasive, safe, successful, and well tolerated. The technique has many advantages over the traditional nasopharyngeal tube and reduces the need for surgical intervention to relieve high upper airway obstruction in infants with craniofacial anomalies.

1 Bull MJ, Givan DC, Sadove AM, Bixler D, Hearn D. Improved outcome in Pierre Robin sequence: effect of multidisciplinary evaluation and management. Pediatrics 1990;86:294-301.

2 Freezer NJ, Beasley SW, Robertson CF. Tracheostomy. Arch Dis Child 1990;65:123-6.

3 Caouette Laberge L, Bayet B, Larocque Y. The Pierre Robin sequence: review of 125 cases and evolution of treatment modalities. Plast Reconstr Surg 1994;93:934-42.

4 Donnelly MJ, Lacey PD, Maguire AJ. A twenty year (19711990) review of tracheostomies in a major paediatric hospital. Int f Pediatr Otorhinolaryngol 1996;35:1-9.

5 Heaf DP, Helms PJ, Dinwiddie R, Matthew DJ. Nasopharyngeal airways in Pierre Robin syndrome. $f$ Pediatr 1982;100:698-703.

6 Shinkwin CA, Gibbin KP. Tracheostomy in children. $\mathscr{F} R$ Soc Med 1996;89:188-92. 